\title{
Chemokine CXCL1-Mediated Neutrophil Trafficking in the Lung: Role of CXCR2 Activation
}

\author{
Kirti V. Sawant ${ }^{a}$ Renling $\mathrm{Xu}^{\mathrm{a}}$ Robert Cox $^{\mathrm{d}}$ Hal Hawkins $^{\mathrm{d}}$ Elena Sbrana $^{\mathrm{d}}$ \\ Deepthi Kollic Roberto P. Garofalo ${ }^{b, c}$ Krishna Rajarathnam ${ }^{a, b, e}$ \\ Departments of a Biochemistry and Molecular Biology, ${ }^{\mathrm{b}}$ Microbiology and Immunology, ${ }^{\mathrm{c} P e d i a t r i c s}$ and ${ }^{\mathrm{d}}$ Pathology, \\ and ' Sealy Center for Structural Biology and Molecular Biophysics, The University of Texas Medical Branch, \\ Galveston, Tex., USA
}

\section{Key Words}

Neutrophils $\cdot$ Receptor signaling $\cdot$ Monomer-dimer equilibrium · Tissue injury

\begin{abstract}
The chemokine CXCL1 and its receptor CXCR2 play a crucial role in host immune response by recruiting and activating neutrophils for microbial killing at the tissue site. Dysregulation in this process has been implicated in collateral tissue damage causing disease. CXCL1 reversibly exists as monomers and dimers, and it has been proposed that distinct monomer and dimer activities and the monomer-dimer equilibrium regulate the neutrophil function. However, the molecular mechanisms linking the CXCL1/CXCR2 axis and the neutrophil 'beneficial' and 'destructive' phenotypes are not known. In this study, we characterized neutrophil trafficking and its consequence in the mouse lung by the CXCL1 wild type (WT), which exists as monomers and dimers, and by a nondissociating dimer. Whereas the WT, compared to the dimer, was more active at low doses, both the WT and the dimer elicited a large neutrophil efflux at high doses. Importantly, robust neutrophil recruitment elicited by the WT or dimer was not detrimental to lung tissue integrity and,
\end{abstract}

further, could not be correlated to surface CXCR2 levels. We conclude that the CXCL1 monomer-dimer distribution and receptor interactions are highly coupled and regulate neutrophil trafficking and that injury in the context of disease is a consequence of inappropriate CXCR2 activation at the target tissue and not due to mechanical forces exerted by neutrophils during recruitment.

(c) 2015 S. Karger AG, Basel

\section{Introduction}

Chemokines, a superfamily of small proinflammatory proteins, play a fundamental role in directing circulating neutrophils to the site of infection and activating them for microbial killing at the tissue site. It is now well established that impaired or excessive neutrophil recruitment or activation results in incomplete resolution or severe collateral tissue damage [1-3]. Several lines of evidence from clinical and animal model studies have shown that the chemokine CXCL1 (MGSA/GROa or keratinocytederived chemokine in the mouse) plays a beneficial role by trafficking and activating neutrophils in response to infections, but is also implicated in exacerbating injury in

\section{KARGER 125}

() 2015 S. Karger AG, Base

$1662-811 \mathrm{X} / 15 / 0076-0647 \$ 39.50 / 0$

E-Mail karger@karger.com

www.karger.com/jin
Dr. Krishna Rajarathnam

Department of Biochemistry and Molecular Biology

5.142 Medical Branch Building, The University of Texas Medical Branch

301 University Boulevard, Galveston, TX 77555-1055 (USA)

E-Mail krrajara@utmb.edu 
various tissues including the lung [4-6]. CXCL1 produced by resident macrophages and/or epithelial cells rapidly travels to the bloodstream, where it orchestrates neutrophil trafficking from vasculature into the tissue [7, 8]. CXCL1 mediates neutrophil recruitment by binding and activating CXCR2, a member of the G protein-coupled receptor class [9]. CXCR2 inhibitor and knockout mice studies have unambiguously shown that a dysregulation in CXCL1/CXCR2 function is correlated with severity of disease [10-13].

Presently, knowledge of the molecular mechanisms that switch the CXCL1-mediated neutrophil function from a 'beneficial' to a 'destructive' phenotype is lacking. Chemokines reversibly exist as monomers and dimers, and it has been proposed that distinct functional properties of the monomers and dimers and of the monomerdimer equilibrium allow the fine-tuning and regulation of neutrophil recruitment from circulation and its activation for microbial killing at the target site $[14,15]$. This is particularly relevant for inflammatory chemokines, such as CXCL1, whose production is transiently upregulated, and so its local concentration can increase by orders of magnitude. We recently made a novel observation that the CXCL1 dimer is as potent as the monomer for various CXCR2 activities [16]. This observation is striking, as the monomer has been shown to be the high-affinity receptor agonist for most other chemokines [16-20]. CXCR2 activation leads to a series of downstream $G$ protein- and $\beta$-arrestin-mediated signaling events that orchestrate adhesion and transmigration across the endothelium, crawling across the extracellular matrix, and finally adhesion and transmigration across the epithelium into the lumen $[7,8,21]$. Chemokine binding also results in $\beta$-arrestinmediated CXCR2 internalization, but occurs at concentrations higher than $G$ protein-/ $\beta$-arrestin-coupled signaling events [22-25]. Therefore, these various receptormediated events are directly dependent on the monomer-dimer ratio and local CXCL1 concentration, and a dysregulation in any of these processes could switch the neutrophil phenotype.

In this study, we tested this hypothesis by characterizing neutrophil levels and the consequence of neutrophil recruitment at various doses of CXCL1 wild type (WT), which exists as monomers and dimers, and of a trapped dimer in the mouse lung. Our data show that the mechanical forces exerted by neutrophils recruited by either the WT or the dimer did not cause tissue damage and, further, could not be correlated to CXCR2 surface levels. We conclude that the CXCL1 monomer-dimer equilibrium and receptor interactions are highly coupled and regulate neutrophil trafficking and that the destructive phenotype manifested in the context of disease is a consequence of inappropriate CXCR2 activation at the target tissue and not due to mechanical forces exerted by neutrophils during recruitment.

\section{Materials and Methods}

\section{Animals}

Female, 7-8-week-old BALB/c mice (Harlan, Houston, Tex., USA) were maintained in specific pathogen-free conditions in the animal research facility of the University of Texas Medical Branch (UTMB), in accordance with the institutional guidelines for animal care of the National Institutes of Health (NIH) and UTMB. Cages, bedding, food, and water were sterilized before use. All animal work was approved by the institutional animal care and use committee.

\section{Design and Synthesis of Human CXCL1 Variants}

The human recombinant CXCL1 WT and trapped N27C dimer were produced as described previously [16]. Briefly, CXCL1 variants were cloned in a pET32 Xa vector and expressed as thioredoxin fusion proteins with a His-tag in the Escherichia coli BL21 (DE3) strain. Transformed E.coli BL21 (DE3) cells were grown to an $A 600$ of 0.8 and induced with $1 \mathrm{mM}$ isopropyl $\beta$-Dthiogalactopyranoside for $8 \mathrm{~h}$ at $37^{\circ} \mathrm{C}$. The cells were grown at $25^{\circ} \mathrm{C}$ in the shaker for another $12-16 \mathrm{~h}$ before harvesting. The proteins were purified using standard Ni-NTA affinity chromatography and reverse-phase high-pressure liquid chromatography. The CXCL1 R8K mutant was generated by PCR amplification using the Quikchange site-directed mutagenesis kit (Stratagene) and were expressed and purified using the same protocol as described above. The purity and molecular weight of the proteins were confirmed using matrix-assisted laser desorption/ionization mass spectrometry (MALDI-MS) and SDS-PAGE.

Recruitment of Neutrophils in Mouse Bronchoalveolar Lumen

The bronchoalveolar lavage was done according to previously published protocols [14]. The mice were intranasally instilled with different doses $(0.1,1$, and $10 \mu \mathrm{g})$ of CXCL1 variants in $50 \mu \mathrm{l}$ of Dulbecco's phosphate-buffered saline (Cellgro, Va., USA) under light anesthesia. After 6 or 24 h of postinstillation of CXCL1 variants, the mice were euthanized by an intraperitoneal injection of a ketamine and xylazine mixture. The bronchoalveolar lavage fluid (BALF) was harvested, spun down, and supernatants were collected. The cell pellet was resuspended in Dulbecco's phosphate-buffered saline, and $100 \mu \mathrm{l}$ of cell suspension were used for the preparation of cytospin slides (Shandon, Thermo Electron Corporation). The slides were fixed and stained with Wright-Giemsa stain for a differential leukocyte count. The leukocyte numbers were measured using a hemocytometer with Turk's diluting fluid (Ricca Chemicals, Tex., USA).

\section{Isolation of Peripheral Blood Leukocytes}

Immediately after euthanasia, blood was drawn from the femoral artery and transferred to Eppendorf tubes containing heparin. The samples were centrifuged at 530 relative centrifugal force for 
3 min at $10^{\circ} \mathrm{C}$, and cell pellets were then lysed using ACK lysing buffer at room temperature. The cells were then washed 2-3 times with PBS, resuspended, and counted using a hemocytometer.

\section{Isolation of Lung Digest Cells}

The lungs of mice treated with CXCL1 variants were harvested after the removal of the BALF. The lungs were cut into small pieces and incubated with $5 \mathrm{ml}$ of Hanks' balanced saline solution buffer containing $0.5 \mathrm{mg} / \mathrm{ml}$ of collagenase A (Sigma) and $0.15 \mathrm{kU}$ of type IV bovine pancreatic DNAase (Sigma) for $1 \mathrm{~h}$ at $37^{\circ} \mathrm{C}$ with intermittent shaking. After digestion, the cells were passed 3-4 times through a 20 -gauge needle and filtered through a $40-\mu \mathrm{m}$ cell strainer to obtain a single-cell suspension. Contaminating red blood cells were lysed using ACK lysing buffer, and the lung cells were then washed with PBS and counted using a hemocytometer.

\section{FACS Analysis}

The BALF, lung, or peripheral blood leukocytes cell $\left(1 \times 10^{6}\right)$ were first incubated with Fc Block (BD Biosciences) for $20 \mathrm{~min}$. For the detection of CXCR2 on neutrophils, the cells were washed with PBS containing EDTA and 1\% FBS, stained with Gr-1 FITC (clone: RB6-8C5), CD11bPerCPCy5.5 (clone: M1/70) (both from BD Biosciences), and CXCR2-PE (clone: 242216) (R\&D systems) and incubated in the dark for $30 \mathrm{~min}$ at $4{ }^{\circ} \mathrm{C}$. The corresponding isotype antibodies were used as controls. The cells were then washed twice and fixed using $2 \%$ paraformaldehyde. The cells were acquired with BD FACS Canto (BD Biosciences) and analyzed using the FlowJo software.

\section{CXCR2 Endocytosis}

CXCR2 endocytosis was measured using differentiated HL60 cells as described previously [26]. Briefly, HL60 cells were induced to differentiate into the neutrophilic lineage [16], and the differentiated cells were incubated with serum-free media for $1 \mathrm{~h}$ at $37^{\circ} \mathrm{C}$ and then resuspended in Hanks' balanced saline solution at $1 \times$ $10^{7} / \mathrm{ml}$. The cells $\left(1 \times 10^{6}\right)$ were then incubated with CXCL1 WT or dimer at different concentrations and time points at $37^{\circ} \mathrm{C}$, washed with ice-cold FACS buffer and then incubated with PEconjugated CXCR2 antibody (clone: 48311 ; R\&D systems) for the detection of the cell surface CXCR2 levels by flow cytometry.

\section{Immunohistochemistry of Lung Sections}

The lungs were collected at necropsy and placed in 10\% buffered formalin for processing and subsequent microscopy. After paraffin embedding, 4- $\mu \mathrm{m}$ sections were stained by the hematoxylin and eosin (H\&E) method for microscopic examination. Unstained sections were deparaffinized using xylene baths and prepared for immunohistochemical staining with anti-Ly6G (BD Biosciences) as primary antibody $(1: 2,000)$ or MPO antibody $(1: 100)$ using the peroxidase method and $\mathrm{DAB}$ as the chromogen. The immunohistochemical sections were examined, and images of 5 random high-power fields (HPFs) were captured from each slide and processed using the Image Pro Plus software (Media Cybernetics) to obtain absolute counts of neutrophils per HPF.

\section{Transmission Electron Microscopy of Lung Sections}

The tissues were collected from control PBS-, WT-, and dimertreated mice. Briefly, lung tissue was minced into $\sim 1 \mathrm{~mm}^{3}$ pieces and placed in $2 \%$ glutaraldehyde in $80 \mathrm{~mm}$ cacodylate buffer, $\mathrm{pH}$ 7.4. Following primary fixation, the tissue was washed and post- fixed with $1 \%$ osmium tetroxide followed by dehydration and embedding in Spurr epoxy resin. Semi-thin sections were cut and stained with $1 \%$ toluidine blue. The examination of the semi-thin sections showed absence of alveolar neutrophils in the control animals and focal areas with alveolar neutrophils in both the WTand the dimer-treated mice. From each mouse, 1 representative block was selected for further transmission electron microscopy (TEM) analysis. Thin sections were stained with uranyl acetate and lead citrate. To assess the tissue morphology, low magnification micrographs (magnification $\times 2,000-3,600$ ) were acquired for virtually all tissues present in the open grid spaces.

\section{Statistics}

Analysis of variance (ANOVA) was used to determine the statistical significance of differences between the variants by using Tukey's post hoc analysis. The statistical tests were performed using the Graph Pad Prism 5 software.

\section{Results}

CXCL1-Mediated Neutrophil Recruitment in the Lung Different doses $(0.1,1$, and $10 \mu \mathrm{g})$ of CXCL1 WT or trapped dimer were intranasally administered in mice, and the BALF was collected after $6 \mathrm{~h}$. Mice treated with PBS served as the control. Both CXCL1 variants were active compared to the control, but the neutrophil levels were markedly different and varied with the dose (fig. 1). CXCL1 WT showed increased recruitment with increasing dose, and significantly higher neutrophil levels were observed at the 1- and 10- $\mu \mathrm{g}$ dose compared to the $0.1-\mu \mathrm{g}$ dose. The dimer was only marginally active at lower doses but as active as the WT at the $10-\mu \mathrm{g}$ dose. In order to rule out any spurious bacterial contamination such as LPS as a source of the recruitment, a CXCL1 variant containing a mutation in the ELR motif (R8K) was prepared using the same protocol as the WT. This mutant was inactive at the 10- $\mu$ g dose. We also determined endogenous chemokine and cytokine concentrations and observed no differences compared to the control (data not shown), indicating that all of the recruitment can be directly attributed to the exogenously administered CXCL1 variants.

CXCL1 variants administered intranasally migrate to the vasculature and direct neutrophil migration from the blood into the lung tissue. Neutrophil trafficking involves 3 distinct steps: traversing the endothelial layer from the vasculature, crawling across the interstitium that consists of matrix proteins and proteoglycans, and finally crossing the epithelial layer to enter the bronchoalveolar lumen. At the 10- $\mu$ g dose, the neutrophil levels recruited via the WT and the dimer were similar both in the lumen (as measured by the BALF) and lung tissue (fig. $2 a$ and $b$ ). 


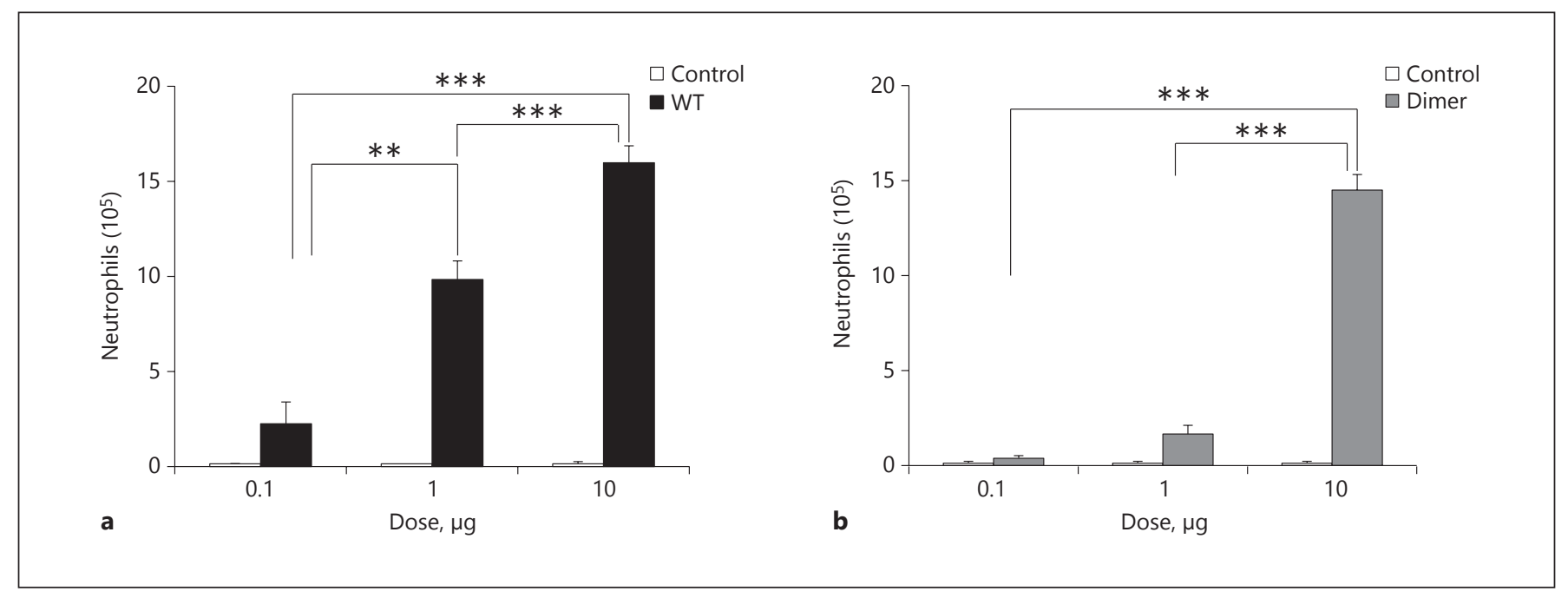

Fig. 1. Neutrophil recruitment profiles of CXCL1 WT and trapped dimer. The BALB/c mice were intranasally administered with 0.1 , 1 , or $10 \mu \mathrm{g}$ of CXCL1 variants, and PBS was used as a control. After $6 \mathrm{~h}$, the BALF cells were harvested, and a leukocyte differential count was performed. The results are expressed as means \pm standard error from 3 independent experiments using 4 animals/ group. The levels of neutrophils are compared between all 3 doses for each variant. ${ }^{* *} \mathrm{p}<0.01,{ }^{* * *} \mathrm{p}<0.001$.
The neutrophil levels were significantly higher in the lung tissue compared to the lumen for both variants. The blood neutrophil levels for both variants were similar compared to the control (fig. 2c), indicating that homeostatic mechanisms most likely replenish the migrated neutrophils with those from the bone marrow in the time frame of the experiment. We also measured the neutrophil levels after the administration at $24 \mathrm{~h}$ and found similar neutrophil numbers for the WT and the dimer but significantly lower levels when compared to $6 \mathrm{~h}$ (fig. $2 \mathrm{~d}$ ).

\section{Neutrophil Localization and Tissue Integrity}

In order to characterize the tissue integrity and identify the mode and sites where neutrophils migrated into the tissue, we characterized lung cross sections from mice treated with the 10- $\mu$ g dose of the CXCL1 WT and the dimer using H\&E staining, immunohistochemistry with different antibodies, TEM, and BALF protein levels.

Immunohistochemistry. The paraffin-embedded lung sections were immunostained with neutrophil-specific Ly6G or myeloperoxidase (MPO) antibodies. Both sections showed similar characteristics (fig. 3 and 4), so we will confine our discussion to Ly6G staining and refer to the MPO staining whenever necessary. At the 6-h time point, neutrophil counts in the WT- and dimer-treated sections were $\sim 100$-fold higher compared to the PBS control. Neutrophil localization was prominent in smaller airways and alveoli and less frequent around large airways and blood vessels. Numerous neutrophils were observed in the airspaces in close proximity to the alveolar epithelial cells and underlying capillaries, and possibly in the interstitium (fig. 3b and c). Neutrophils were rarely observed around the major peribronchiolar or perivascular areas, which suggests that the exogenously administered chemokine travels to the alveoli from where it migrates to the underlying capillaries and orchestrates neutrophil migration to the bronchoalveolar lumen. Both the WTand the dimer-treated lungs showed multifocal and diffuse neutrophilic infiltrates in the lung parenchyma. Compared to the 6-h time point, fewer but similar amounts of neutrophils were observed for the 24-h time point for the WT and the dimer (fig. 3d and e). Most importantly, there was also no evidence of tissue damage at the 24-h time point. Further, quantitative analysis at both the 6-h and the 24-h time point showed higher neutrophil counts per HPF in the WT- and dimer-treated groups, compared to the sections treated with the PBS control (fig. $3 \mathrm{f}$ and g). The recruitment profiles were similar to those observed from differential cell counts as shown in figure 2. The areas of neutrophil infiltration were not associated with desquamation of the epithelium, and there was no evidence that the integrity of the alveolar wall was compromised. Similar observations were made from the MPO-stained slides (fig. 4). In fact, these sections looked no different from the PBS control mice. We also examined $\mathrm{H} \& \mathrm{E}$ staining of the lung cross sections and observed 


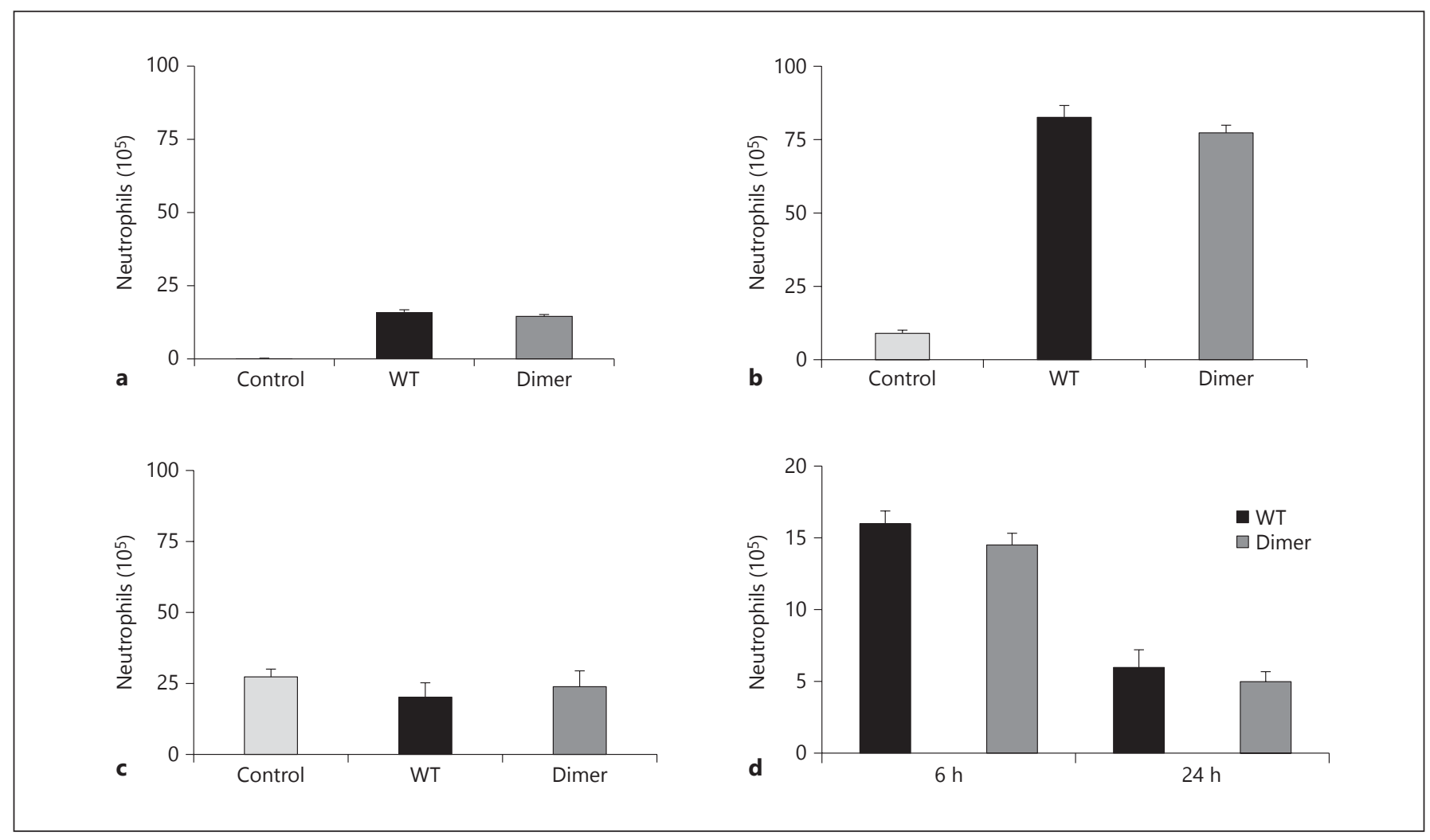

Fig. 2. Neutrophil levels in various compartments. The BALBc mice were intranasally administered with $10 \mu \mathrm{g}$ of CXCL1 WT or dimer. The BALF, lung, and blood were harvested after $6 \mathrm{~h}$. The recruited neutrophil levels in various compartments are shown. a BALF. b Lung tissue. c Blood. d The neutrophil recruitment of WT versus dimer was compared at the 6 -h and 24 -h time points. Each data set represents an average of 3 independent experiments using 4 animals/group, and the results are expressed as means \pm standard error. no evidence of tissue damage in the chemokine-treated mice compared to the control (data not shown). High neutrophil levels at $6 \mathrm{~h}$, much lower levels at $24 \mathrm{~h}$, and lack of damage at both time points suggest that the 6-h time point corresponds to the active recruitment phase and the 24-h time point to the resolution phase.

Transmission Electron Microscopy. As immunohistochemistry and H\&E staining provide low-resolution images, we also characterized the TEM of the lung cross sections to gain better definition at the individual cell and subcellular levels. We characterized the lung cross sections from mice challenged with WT, trapped dimer, and PBS control $(\mathrm{n}=3)$ for the 6 -h time point with the $10-\mu \mathrm{g}$ dose. In PBS-treated mice, parenchymal morphology appeared normal with open alveolar spaces without exudative material (fig. 5a). The septal walls had no interstitial edema, and an occasional neutrophil was seen in the vascular lumen. No discontinuities in the alveolar epithelium or vascular endothelium were noted. In the WT-challenged animals, neutrophils were present in the alveolar space, vascular lumen, and in the septal interstitium (fig. 5b). No interstitial edema was noted. In many alveolar spaces, a protein exudate was present with occasional dense osmophilic debris consistent with the secretion of type II cell granule content. No disruption in the alveolar epithelium was noted, although focally, type I cells showed some swelling and loss of cytoplasmic density. The swelling of the type I cells was not associated with the presence of neutrophils in the alveolar or interstitial spaces. The capillary endothelial cells showed normal morphology and, in some cases, cytoplasmic swelling with loss of cytoplasmic density. No disruption in the endothelial barrier was noted. The ultrastructure of the dimer-challenged mice was no different from that of the WT-challenged mice (data not shown). Consistent among the samples was the presence of neutrophils in the alveolar space, interstitium, and vascular lumen. Many alveoli showed a protein exudate, although no disruption in the integrity of the alveolar epithelium or capillary endothelium was noted. As with the WT-challenged animals, no 


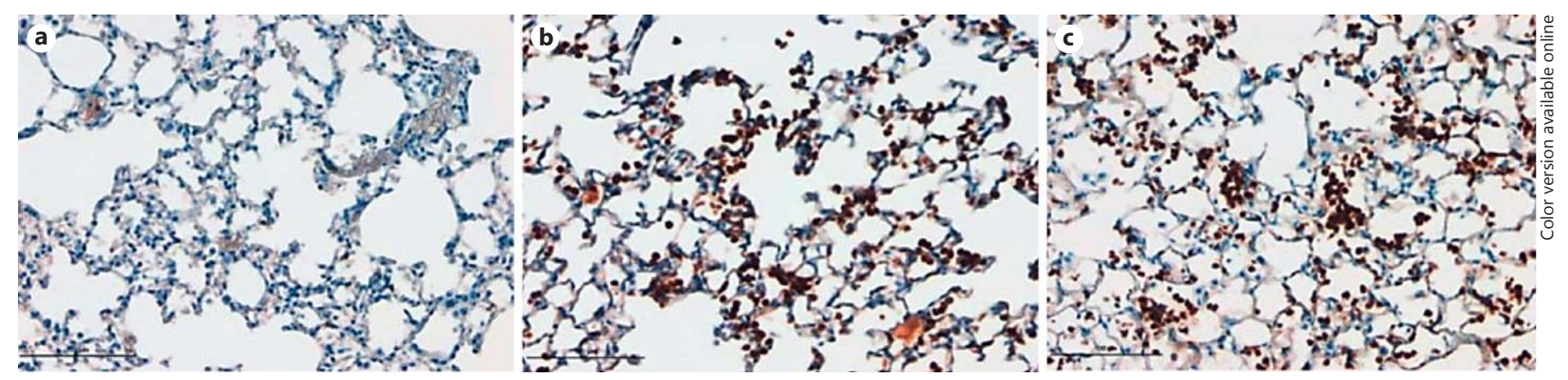

Fig. 3. Ly6G staining of lung sections from CXCL1-treated mice. The lung sections were stained for neutrophils using anti-Ly6G (BD Biosciences) as the primary antibody. Representative lung sections from mice treated with PBS control (a), WT (b), and dimer (c) at the 6-h time points, and with WT (d) and dimer (e) at the 24-h time points are shown. The neutrophil counts captured from 5 random HPFs of lung sections from each slide at the 6-h and 24-h time points are shown in $\mathbf{f}$ and $\mathbf{g}$, respectively. The data are expressed as the means \pm standard error of the results obtained from 2 independent experiments with 4 animals/group. The differences between the variants were compared using ANOVA followed by Tukey's post hoc analysis. ${ }^{*} \mathrm{p}<0.05$, $* * \mathrm{p}<0.01$.
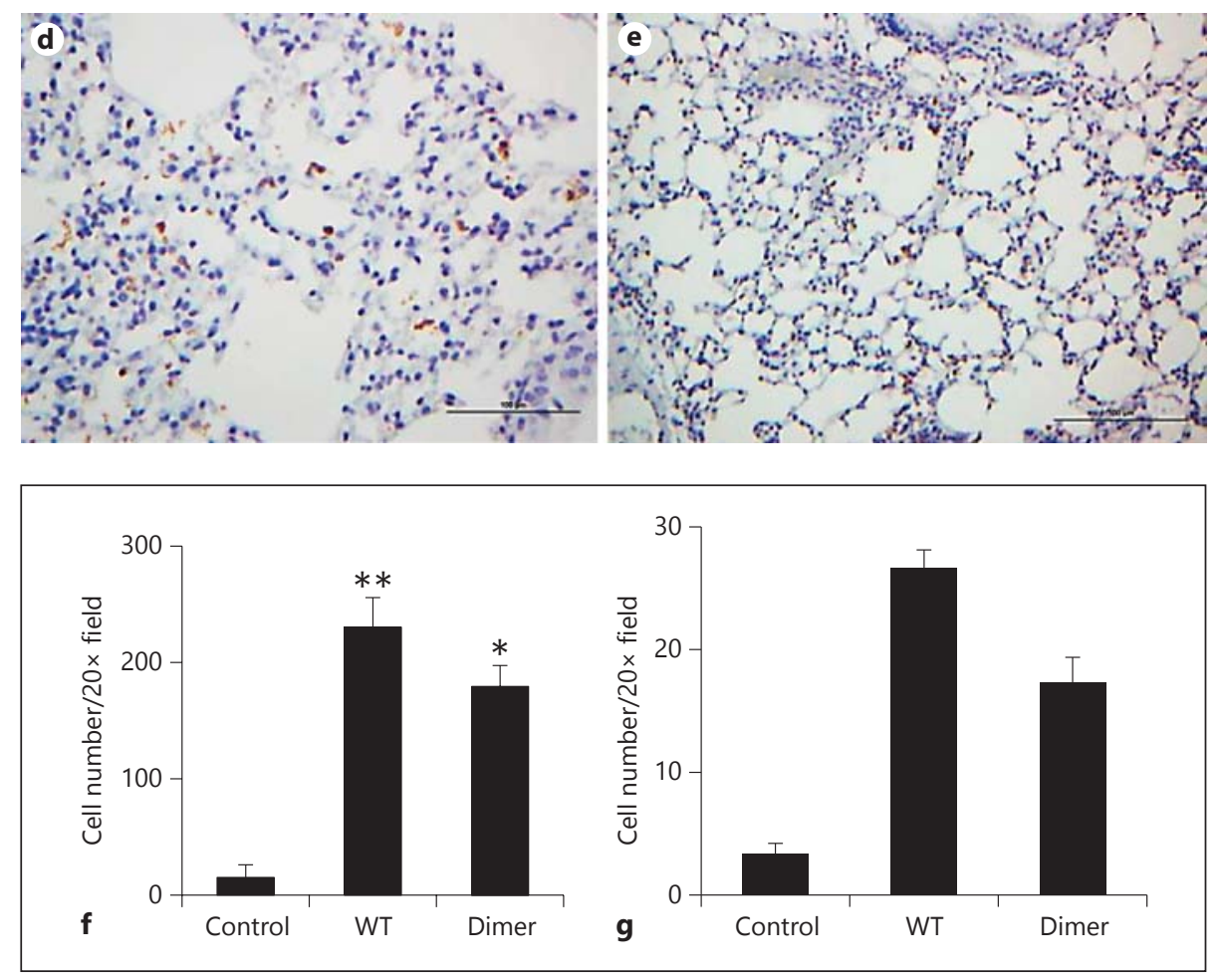

increase in interstitial edema was noted. The TEM analyses collectively indicate that any tissue damage is minimal and that neutrophil trafficking does not disrupt the integrity of the endothelium or epithelium.

BALF Protein Levels. Increased protein levels in the lumen are generally interpreted as a result of damaged epithelium [27]. Therefore, we measured protein levels in the BALF at $6 \mathrm{~h}$ and observed no differences for the dimer and a $\sim 2$-fold increase for the WT compared to the control (fig. 6a). We characterized the BAL proteins by SDSPAGE, and observed a prominent band corresponding to albumin in all cases, including from the PBS control (data not shown). We also measured the protein levels for both the WT and the dimer at $24 \mathrm{~h}$ and observed no significant difference between the variants and compared to the con- trol (fig. 6b). The differences in protein levels between $\mathrm{WT}$ and dimer at the 6-h time point must be a consequence of the property of the WT to exist as monomers and dimers, considering that the immunohistochemical and TEM data showed no evidence of damage or any significant difference between WT and dimer.

\section{CXCR2 Surface Levels in Recruited Neutrophils}

In order to understand the relationship between neutrophil recruitment and CXCR2 function, we characterized the CXCR2 levels in the BALF, lung, and blood neutrophils for the $10-\mu \mathrm{g}$ data by gating for the $\mathrm{Gr}-1^{+} / \mathrm{CD} 11 \mathrm{~b}^{+}$ cells in the FSC/SSC subset using FACS analysis (fig. 7). The CXCR2 levels on WT-recruited neutrophils were consistently lower in both BAL and lung though both WT 

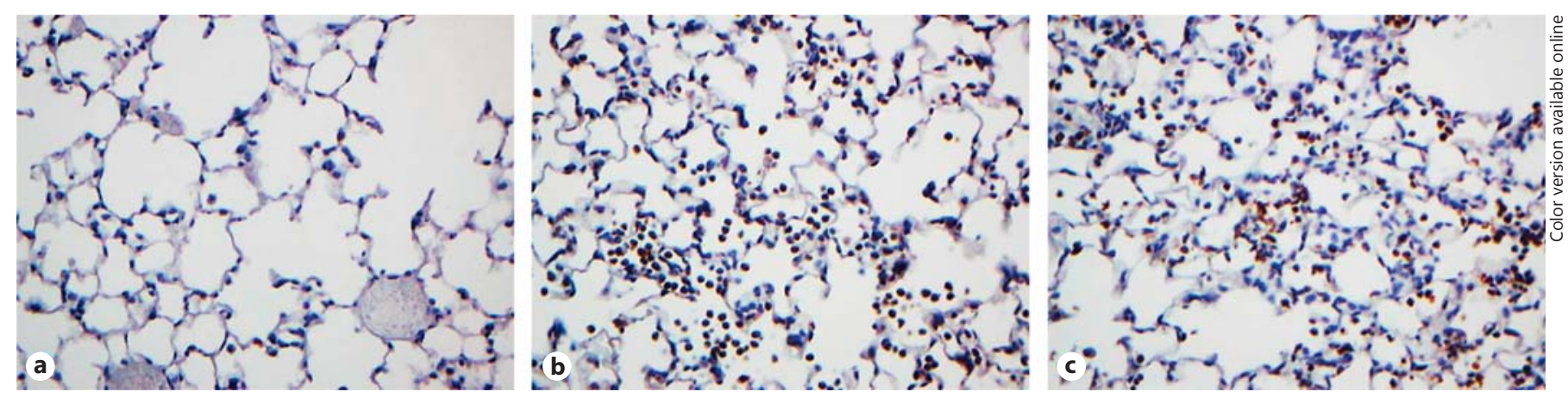

Fig. 4. MPO staining of lung sections from CXCL1-treated mice. The lung sections were stained for neutrophils using anti-MPO (Abcam) as the primary antibody. Representative lung sections from mice treated with PBS control (a), WT (b), and dimer (c) at the 6-h time points are shown.
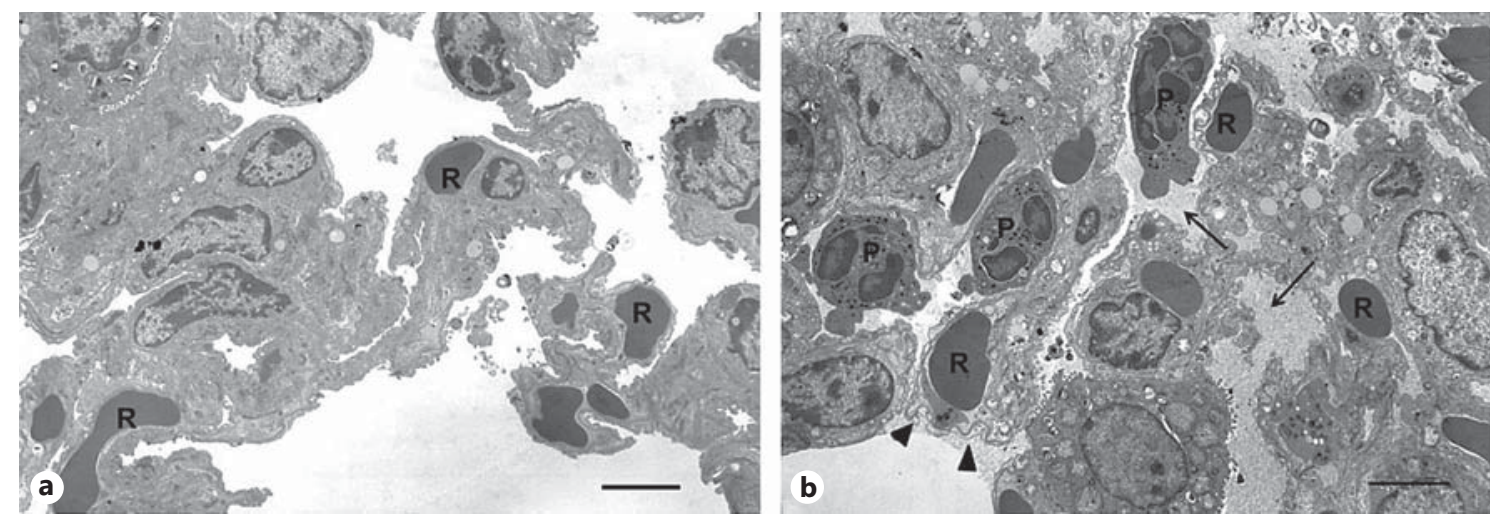

Fig. 5. TEM of lung sections from CXCL1-treated mice. a TEM micrograph of the PBS control showing alveolar spaces without protein exudate or neutrophils. The interstitium of the septum lacks edematous spaces and congestion and has an intact alveolar epithelium and capillary endothelium with red blood cells (R). b TEM micrograph from the WT-treated mouse showing alveolar spaces with a protein exudate (arrows) and neutrophils (P). The septum is uncongested with red blood cells $(\mathrm{R})$ and edematous spaces. The alveolar epithelial type I cells show limited focal swelling and loss of cytoplasmic density (arrow heads) with the vascular endothelium intact. Scale bar $\sim 3 \mu \mathrm{m}$.

deplete the surface CXCR2 levels. We measured the endocytosis rates using CXCR2-expressing HL60 cells and observed that both WT and dimer triggered CXCR2 internalization with similar efficiency (fig. 8). Both WT and dimer showed weak internalization activity at 10 and $50 \mathrm{nM}$ (fig. $8 \mathrm{a}$ and b) and robust internalization at $100 \mathrm{nM}$ (fig. 8c). At later time points, the receptor levels start to recover at similar rates for $\mathrm{WT}$ and dimer $(\sim 60 \%$ at $6 \mathrm{~h})$. Our previous studies have shown that the dimer is a potent agonist for CXCR2 activities such as $\mathrm{Ca}^{2+}$ release and ERK phosphorylation [16]. Therefore, differences in in vivo CXCR2 levels must be a reflection of differences in local CXCL1 concentration, which is most likely due to differences in their glycosaminoglycan (GAG) interactions at different locations along the migration path. In- 


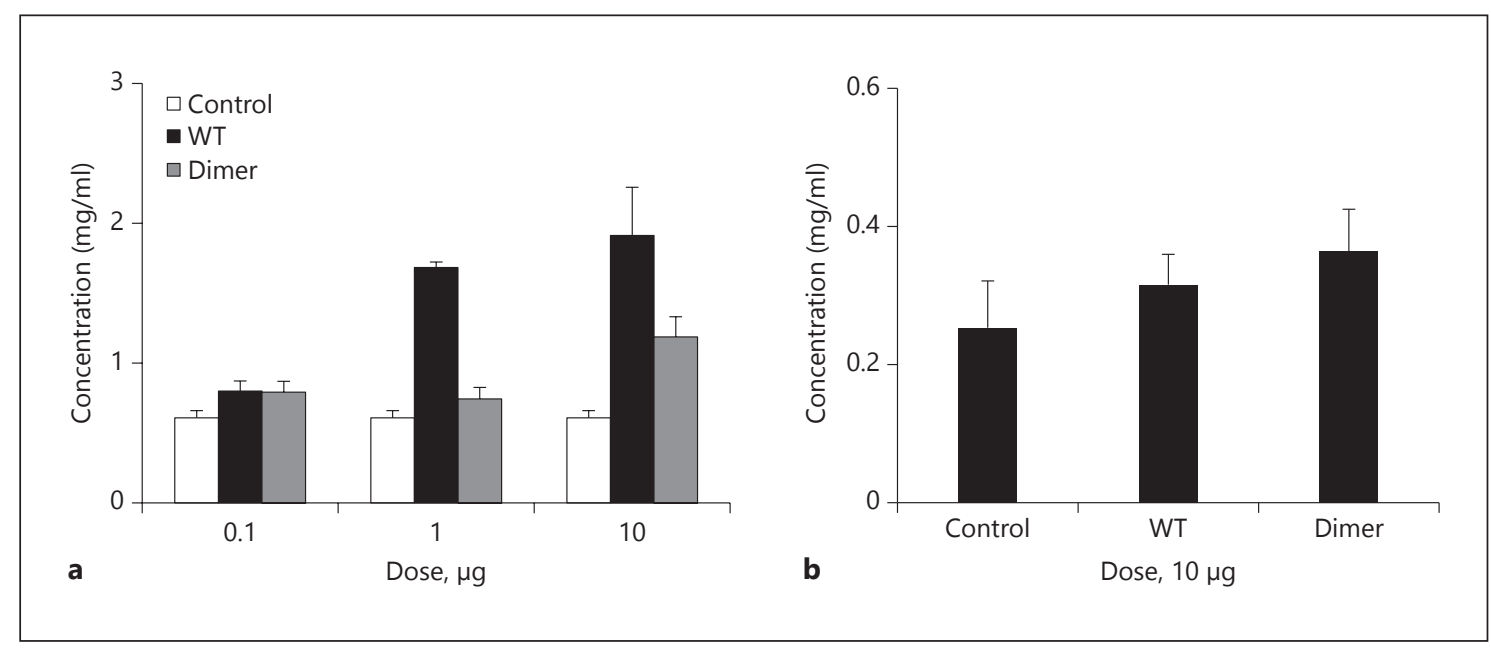

Fig. 6. BALF protein levels from CXCL1-treated mice. Protein levels in the BALF supernatants at both the (a) 6-h and the (b) 24-h time points for the $10-\mu \mathrm{g}$ dose were estimated using the BCA protein assay kit (Thermo Fisher Scientific) as described previously [14]. The data are expressed as the means \pm standard error of the results obtained from 2 independent experiments with 4 animals/group. deed, our in vitro studies have shown that the dimer binds GAG with higher affinity than does the monomer (unpublished results). These observations further indicate that the WT's property of existing as monomers and dimers at higher doses does not influence neutrophil numbers and most likely plays a role in defining the phenotype for microbial killing in the tissue.

\section{Discussion}

In this study, we have characterized the relationship between CXCL1 levels and monomer-dimer equilibrium, CXCR2 levels and their activation, neutrophil levels and trafficking, and tissue damage. During active recruitment, the CXCL1 levels can vary spatially and temporally by many orders of magnitude and can thus exist as a monomer, a dimer, or both. CXCL1 also binds to GAGs, and thus the local chemokine concentrations and dimermonomer ratio will thus also be influenced by the GAGbinding affinities. In addition, CXCL1 can be washed away in the circulation or endocytosed on receptor activation. Therefore, continuous changes in the CXCL1 concentration will result in continuous changes in the monomer-dimer ratio influencing the neutrophil function in health and disease.

Infection or injury triggers a cascade of events including the release of multiple chemokines and other chemotactic factors (such as fMLP and LTB4), which complicate establishing the causal relationships of how a given che- mokine mediates recruitment and activation in the tissue. In principle, each factor could play a prominent role in recruitment, activation, or both. Studies from animal models and cell-based studies have shown that resident macrophages secrete chemokines in the lumen and that epithelial cells release chemokines to both the apical and the basolateral sides of the cell $[5,6,28,29]$. Therefore, our strategy of introducing CXCL1 in the alveolar cavity mimics the in vivo chemokine release and provides a spatiotemporal description of its function without the complexities of the multitude of events that occur during an actual infection.

Both WT and dimer elicit robust neutrophil recruitment at high doses, but WT alone is highly active at lower doses. This provides definitive evidence that the ability of the WT to exist as monomers and dimers is essential for neutrophil trafficking under conditions of low protein concentration. Whereas neutrophils are virtually absent and are dominated by macrophages in the alveolar lumen under basal conditions, neutrophils dominate in chemokine-treated mice ( $~ 90 \%$ of all BALF cells), and their levels were comparable to those seen in infection models [ 30 , 31]. Nevertheless, our data showed no evidence of tissue damage indicating that the mechanics of recruitment are highly coordinated and that neutrophils are not activated to release proteases or reactive oxygen species. We did observe $\sim 2$-fold higher protein levels in WT-recruited neutrophils, but our previous studies with CXCL8, and other studies using leukotriene $\mathrm{B} 4$, have also shown a similar increase in BALF protein levels with no evidence 


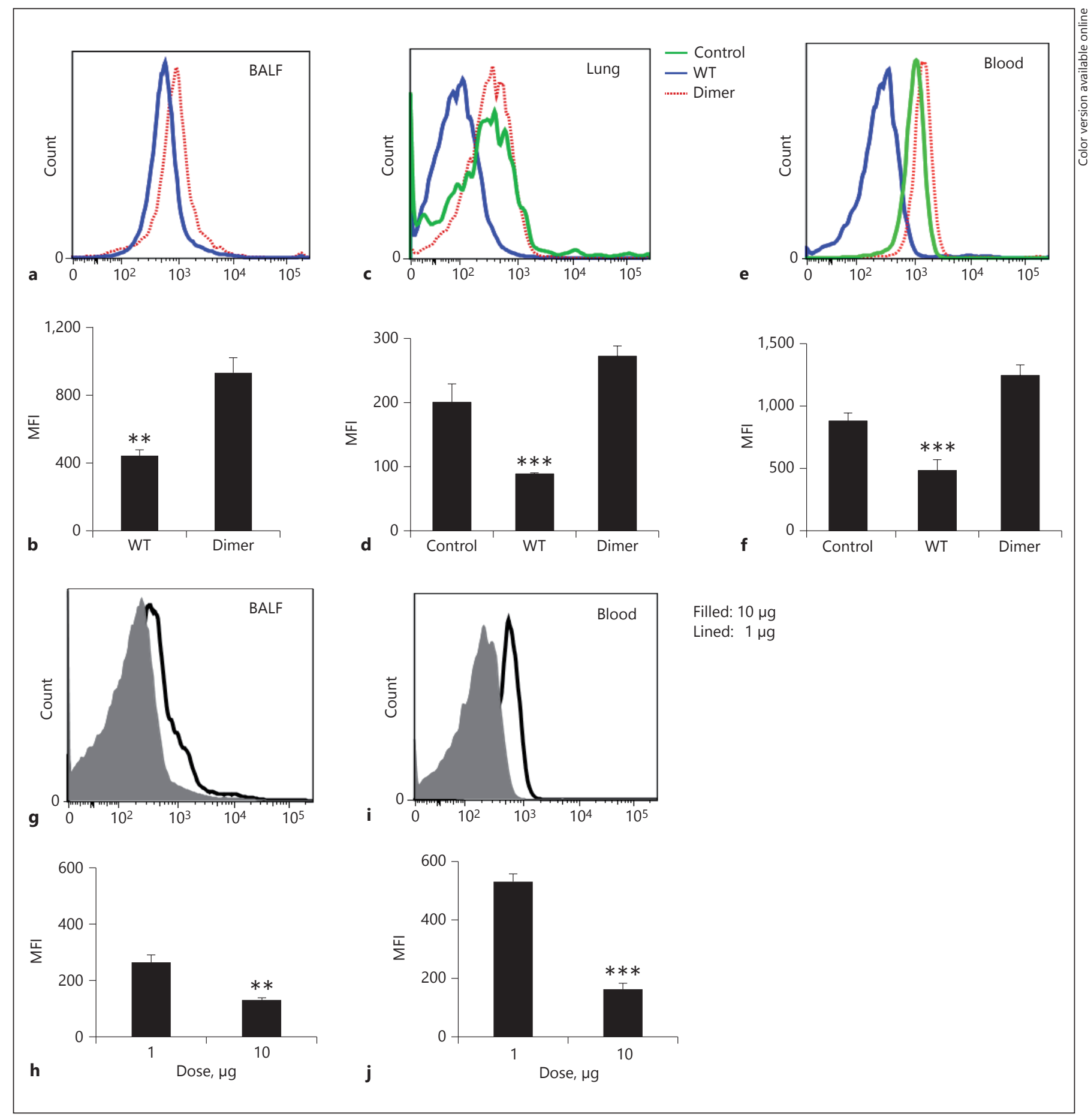

Fig. 7. Surface CXCR2 levels on recruited neutrophils. Leukocytes isolated from BALF, lung, and peripheral blood at the 6-h time point were stained with Gr-1, CD11b, and CXCR2 antibodies, and a FACS analysis was done by gating the neutrophils $\left(\mathrm{Gr}^{+} / \mathrm{CD} 11 \mathrm{~b}^{+}\right)$ in the FSC/SSC subset followed by the detection of the CXCR2 levels. The representative histograms are shown for the BALF (a), lung (c), and blood cells (e). The corresponding bar graphs of the MFI are shown in $\mathbf{b}, \mathbf{d}$, and $\mathbf{f}$, respectively. The CXCR2 levels are compared between the 1- $\mu \mathrm{g}$ and $10-\mu \mathrm{g}$ doses for the CXCL1 WTrecruited neutrophils in the BALF ( $\mathbf{g})$ and blood (i), and the corresponding bar graphs of the MFI are shown in $\mathbf{h}$ and $\mathbf{j}$, respectively. The values are expressed as means \pm standard error, and the differences between the variants were compared using ANOVA followed by Tukey's post hoc analysis. The data shown are representative of 3 independent experiments with 4 animals/group. $\mathrm{MFI}=$ median fluorescence intensity. ${ }^{* *} \mathrm{p}<0.01,{ }^{* * *} \mathrm{p}<0.001$. 


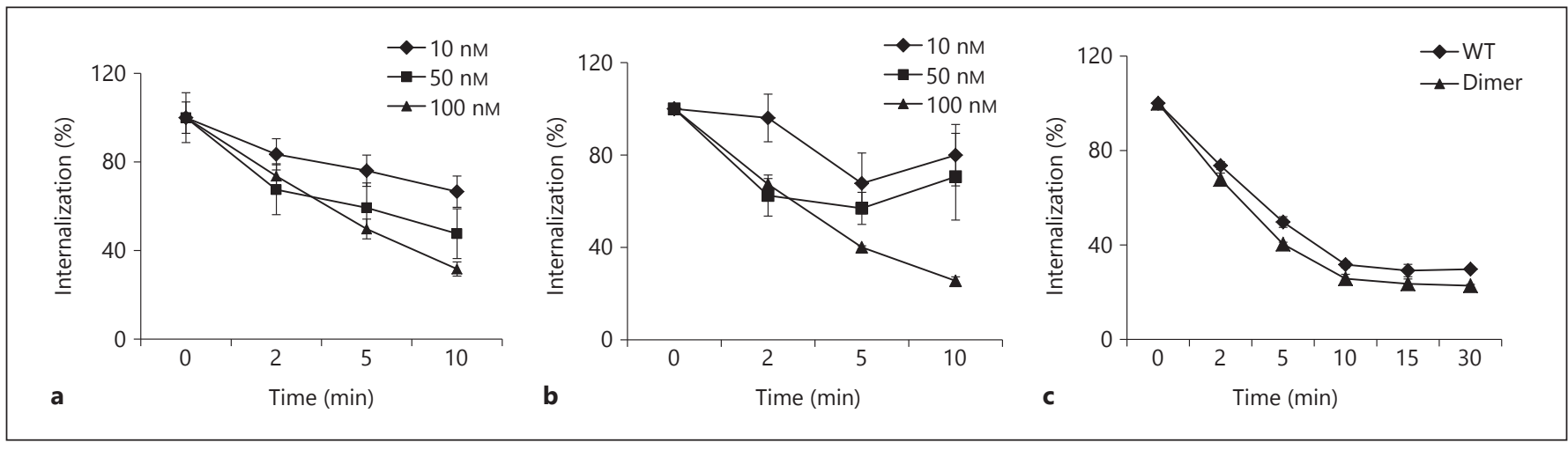

Fig. 8. Endocytosis of CXCR2. HL60 cells stably transfected with CXCR2 were treated with different doses of WT and dimer, and the internalization at different times was measured by flow cytometry. The data for the WT (a) and dimer (b) at different doses and the internalization for both the WT and the dimer at $100 \mathrm{nM}$ (c) are shown. The data were collected in quadruplicate and are presented as the means \pm standard error of the results, and they are representative of 3 independent experiments. of tissue damage $[14,32]$. The BALF from acute respiratory distress syndrome patients shows much higher protein levels ( $>12$ times), suggesting that the increased protein levels in our studies occur with the transient opening of epithelial and endothelial junctions during neutrophil egress [27]. These observations indicate that the gap junctions are highly plastic and that the molecular machinery in the epithelial and endothelial cells and the extracellular matrix are capable of trafficking large numbers of chemokine-mediated neutrophils in a highly orchestrated manner.

During active recruitment, CXCR2 on neutrophils are continuously exposed to CXCL1 both in the vasculature and the tissue. Cell-based studies have shown that receptor activation is also accompanied by receptor endocytosis and that the internalized receptor is trafficked to the lysosome $[23,33]$. The CXCR2 levels in WT-recruited neutrophils were consistently lower, but nevertheless showed no correlation to neutrophil levels. Reduced CXCR2 levels have been observed in BALF neutrophils from patients suffering from various pulmonary diseases and also in peripheral, BALF, and lung tissue neutrophils in CXCL1- and LPS-treated mice [34-38]. Whether the lower receptor levels in these neutrophils are due to endocytosis during the process of recruitment or due to CXCR2 activation in the tissue is not known. It is also unknown whether lower receptor levels are the cause or effect of the disease process. Our data do indicate that neutrophil and CXCR2 levels are not correlated, providing some insight into the causative and correlative relationships among CXCL1, CXCR2, neutrophil levels, and tissue damage.
The CXCL1 recruitment profile was distinctly different compared to the related chemokine CXCL8. CXCL1 WT compared to CXCL8 was significantly more active at the $1-\mu \mathrm{g}$ dose but showed similar recruitment at the $10-\mu \mathrm{g}$ dose. For both CXCL1 and CXCL8, the trapped dimer showed impaired neutrophil recruitment at low doses, but CXCL8 was more active at the higher dose [14]. Whereas CXCL1 and CXCL8 dimers undergo endocytosis like their WT monomers, their receptor activities differ. The CXCL8 dimer is differentially active showing similar or lower activity, but the CXCL1 dimer is highly active $[16,17,39]$. Therefore, differences in recruitment between CXCL8 and CXCL1 and between monomers and dimers are unlikely due to differences in receptor endocytosis and most likely due to differences in receptor activity and/or GAG interactions.

In summary, our study provides compelling evidence that the molecular mechanisms underlying CXCL1- and CXCR2-mediated in vivo neutrophil recruitment are highly orchestrated, that the rapid migration of large levels of neutrophils across various barriers does not cause tissue damage, and that the CXCL1 monomer-dimer equilibrium and distinct activities of monomers and dimers most likely play differential spatiotemporal roles during recruitment and microbial killing.

\section{Acknowledgments}

We thank the University of Texas Medical Branch (UTMB) histochemistry core for the immunohistochemistry, the UTMB FACS core and Kimberley Palkowetz for the flow cytometry, and Shriner's Hospital Electron Microscopy laboratory for the TEM. 
We thank Dr. Jiqing Sai of the Vanderbilt University for the HL60 cells, Dr. Krishna Mohan Poluri for the R8K mutant, and Meenakshi Shanmugasundaram for the technical assistance. This work was supported in part by grants from the National Institutes of Health (P01 HL107152 and R01 AI069152) to K.R.

\section{Disclosure Statement}

The authors declare to have no conflicts of interest.

\section{References}

1 Kolaczkowska E, Kubes P: Neutrophil recruitment and function in health and inflammation. Nat Rev Immunol 2013;13:159-175.

2 Grommes J, Soehnlein O: Contribution of neutrophils to acute lung injury. Mol Med 2011;17:293-307.

3 Reutershan J, Ley K: Bench-to-bedside review: acute respiratory distress syndrome how neutrophils migrate into the lung. Crit Care 2004;8:453-461.

4 Strieter RM, Keane MP, Burdick MD, Sakkour A, Murray LA, Belperio JA: The role of CXCR2/CXCR2 ligands in acute lung injury. Curr Drug Targets Inflamm Allergy 2005;4: 299-303.

5 Balamayooran G, Batra S, Fessler MB, Happel KI, Jeyaseelan S: Mechanisms of neutrophil accumulation in the lungs against bacteria. Am J Respir Cell Mol Biol 2010;43:5-16.

6 Bhatia M, Zemans RL, Jeyaseelan S: Role of chemokines in the pathogenesis of acute lung injury. Am J Respir Cell Mol Biol 2012;46: 566-572.

7 Ley K, Laudanna C, Cybulsky MI, Nourshargh S: Getting to the site of inflammation: the leukocyte adhesion cascade updated. Nat Rev Immunol 2007;7:678-689.

8 Phillipson M, Heit B, Colarusso P, Liu L, Ballantyne CM, Kubes P: Intraluminal crawling of neutrophils to emigration sites: a molecularly distinct process from adhesion in the recruitment cascade. J Exp Med 2006;203:25692575.

9 Ahuja SK, Murphy PM: The CXC chemokines growth-regulated oncogene (GRO) alpha, GRObeta, GROgamma, neutrophil-activating peptide-2, and epithelial cell-derived neutrophil-activating peptide-78 are potent agonists for the type $\mathrm{B}$, but not the type $\mathrm{A}$, human interleukin-8 receptor. J Biol Chem 1996;271:20545-20550.

10 Nagarkar DR, Wang Q, Shim J, Zhao Y, Tsai WC, Lukacs NW, Sajjan U, Hershenson MB: CXCR2 is required for neutrophilic airway inflammation and hyperresponsiveness in a mouse model of human rhinovirus infection. J Immunol 2009;183:66986707.

11 Chapman RW, Phillips JE, Hipkin RW, Curran AK, Lundell D, Fine JS: CXCR2 antagonists for the treatment of pulmonary disease. Pharmacol Ther 2009;121:55-68.

12 Herbold W, Maus R, Hahn I, Ding N, Srivastava M, Christman JW, Mack M, Reutershan J, Briles DE, Paton JC, Winter C, Welte T, Maus UA: Importance of CXC chemokine re- ceptor 2 in alveolar neutrophil and exudate macrophage recruitment in response to pneumococcal lung infection. Infect Immun 2010; 78:2620-2630.

13 Stillie R, Farooq SM, Gordon JR, Stadnyk AW: The functional significance behind expressing two IL-8 receptor types on PMN. J Leukoc Biol 2009;86:529-543.

14 Das ST, Rajagopalan L, Guerrero-Plata A, Sai J, Richmond A, Garofalo RP, Rajarathnam K: Monomeric and dimeric CXCL8 are both essential for in vivo neutrophil recruitment. PLoS One 2010;5:e11754.

15 Gangavarapu P, Rajagopalan L, Kolli D, Guerrero-Plata A, Garofalo RP, Rajarathnam $\mathrm{K}$ : The monomer-dimer equilibrium and glycosaminoglycan interactions of chemokine CXCL8 regulate tissue-specific neutrophil recruitment. J Leukoc Biol 2012;91:259-265.

16 Ravindran A, Sawant KV, Sarmiento J, Navarro J, Rajarathnam K: Chemokine CXCL1 dimer is a potent agonist for the CXCR2 receptor. J Biol Chem 2013;288:12244-12252.

17 Rajarathnam K, Kay CM, Dewald B, Wolf M, Baggiolini M, Clark-Lewis I, Sykes BD: Neutrophil-activating peptide-2 and melanoma growth-stimulatory activity are functional as monomers for neutrophil activation. J Biol Chem 1997;272:1725-1729.

18 Jin H, Shen X, Baggett BR, Kong X, Li-Wang PJ: The human CC chemokine MIP-1beta dimer is not competent to bind to the CCR5 receptor. J Biol Chem 2007;282:2797627983.

19 Drury LJ, Ziarek JJ, Gravel S, Veldkamp CT, Takekoshi T, Hwang ST, Heveker N, Volkman BF, Dwinell MB: Monomeric and dimeric CXCL12 inhibit metastasis through distinct CXCR4 interactions and signaling pathways. Proc Natl Acad Sci U S A 2011;108:1765517660.

20 Rajarathnam K, Prado GN, Fernando H, Clark-Lewis I, Navarro J: Probing receptor binding activity of interleukin-8 dimer using a disulfide trap. Biochemistry 2006;45:78827888.

21 Sadik CD, Kim ND, Luster AD: Neutrophils cascading their way to inflammation. Trends Immunol 2011;32:452-460.

22 Molteni R, Crespo CL, Feigelson S, Moser C, Fabbri M, Grabovsky V, Krombach F, Laudanna C, Alon R, Pardi R: Beta-arrestin 2 is required for the induction and strengthening of integrin-mediated leukocyte adhesion during CXCR2-driven extravasation. Blood 2009; 114:1073-1082.
23 Rose JJ, Foley JF, Murphy PM, Venkatesan S: On the mechanism and significance of ligand-induced internalization of human neutrophil chemokine receptors CXCR1 and CXCR2. J Biol Chem 2004;279:2437224386.

24 Richardson RM, Marjoram RJ, Barak LS, Snyderman R: Role of the cytoplasmic tails of CXCR1 and CXCR2 in mediating leukocyte migration, activation, and regulation. J Immunol 2003;170:2904-2911.

25 Yang W, Wang D, Richmond A: Role of clathrin-mediated endocytosis in CXCR2 sequestration, resensitization, and signal transduction. J Biol Chem 1999;274:11328-11333.

26 Meiser A, Mueller A, Wise EL, McDonagh EM, Petit SJ, Saran N, Clark PC, Williams TJ, Pease JE: The chemokine receptor CXCR3 is degraded following internalization and is replenished at the cell surface by de novo synthesis of receptor. J Immunol 2008;180:67136724.

27 Holter JF, Weiland JE, Pacht ER, Gadek JE, Davis WB: Protein permeability in the adult respiratory distress syndrome. Loss of size selectivity of the alveolar epithelium. J Clin Invest 1986;78:1513-1522.

28 Chan MC, Chan RW, Yu WC, Ho CC, Chui WH, Lo CK, Yuen KM, Guan YI, Nicholls JM, Peiris JS: Influenza H5N1 virus infection of polarized human alveolar epithelial cells and lung microvascular endothelial cells. Respir Res 2009;10:102.

29 Oshansky CM, Barber JP, Crabtree J, Tripp RA: Respiratory syncytial virus $\mathrm{F}$ and $\mathrm{G}$ proteins induce interleukin lalpha, CC, and CXC chemokine responses by normal human bronchoepithelial cells. J Infect Dis 2010;201: 1201-1207.

30 Tate MD, Deng YM, Jones JE, Anderson GP, Brooks AG, Reading PC: Neutrophils ameliorate lung injury and the development of severe disease during influenza infection. J Immunol 2009; 183:7441-7450.

31 Zhang S, Zhang S, Garcia-Vaz E, Herwald H, Gomez MF, Thorlacius H: Streptococcal M1 protein triggers chemokine formation, neutrophil infiltration, and lung injury in an NFAT-dependent manner. J Leukoc Biol 2015, Epub ahead of print.

32 Martin TR, Pistorese BP, Chi EY, Goodman RB, Matthay MA: Effects of leukotriene B4 in the human lung. Recruitment of neutrophils into the alveolar spaces without a change in protein permeability. J Clin Invest 1989;84: 1609-1619. 
33 Samanta AK, Oppenheim JJ, Matsushima K: Interleukin 8 (monocyte-derived neutrophil chemotactic factor) dynamically regulates its own receptor expression on human neutrophils. J Biol Chem 1990;265:183-189.

34 Belperio JA, Keane MP, Burdick MD, Londhe V, Xue YY, Li K, Phillips RJ, Strieter RM: Critical role for CXCR2 and CXCR2 ligands during the pathogenesis of ventilator-induced lung injury. J Clin Invest 2002;110:17031716.

35 Cummings CJ, Martin TR, Frevert CW, Quan JM, Wong VA, Mongovin SM, Hagen TR,
Steinberg KP, Goodman RB: Expression and function of the chemokine receptors CXCR1 and CXCR2 in sepsis. J Immunol 1999;162: 2341-2346.

36 Quaid GA, Cave C, Robinson C, Williams MA, Solomkin JS: Preferential loss of CXCR2 receptor expression and function in patients who have undergone trauma. Arch Surg 1999; 134:1367-1371.

37 Tarlowe MH, Duffy A, Kannan KB, Itagaki K, Lavery RF, Livingston DH, Bankey P, Hauser CJ: Prospective study of neutrophil chemokine responses in trauma patients at risk for pneumonia. Am J Respir Crit Care Med 2005; 171:753-759.

38 Von Vietinghoff S, Asagiri M, Azar D, Hoffmann A, Ley K: Defective regulation of CXCR2 facilitates neutrophil release from bone marrow causing spontaneous inflammation in severely NF-kappa B-deficient mice. J Immunol 2010;185:670-678

39 Nasser MW, Raghuwanshi SK, Grant DJ, Jala VR, Rajarathnam K, Richardson RM: Differential activation and regulation of CXCR1 and CXCR2 by CXCL8 monomer and dimer. J Immunol 2009;183:3425-3432. 